occur on the limb of Jupiter at the latitude of the expected impact site. The two short flashes are probably associated with the initial meteor streak and the subsequent fireball, respectively. The bright flare occurs when the impact site rotates into view. If this interpretation is correct then fragment $\mathrm{R}$ collided with Jupiter at 05:34:45 UT.

Our observations suggest the plume to reach altitudes maybe many hundreds of kilometers above the visible cloud tops. The luminosity of the impact site during the long bright flare is probably maintained by the release of gravitational potential energy as plume material falls back onto the lower atmosphere. Our results agree qualitatively with the predictions for the explosion of $\mathrm{a} \approx 0.5 \mathrm{~km}$ diameter comet fragment which releases $\approx 10^{27} \mathrm{ergs}$ in the Jovian atmosphere at a depth corresponding to a pressure of about 2 bars.

\title{
NEAR-IR SPECTROSCOPIC MONITORING OF JUPITER AT THE TIME OF THE SL-9 IMPACT
}

R. Schulz (MPAE, Germany), Th. Encrenaz (Obs. Paris, France), J.A. Stüwe (MPAE, Germany), G. Wiedemann (ESO)

Jupiter was monitored spectroscopically in the near-IR with the IRSPEC imaging spectrograph mounted on the $3.5 \mathrm{~m}$ New Technology Telescope at ESO, La Silla, Chile, between 16 and 28 July 1994 (except for 19 to 21 July due to bad weather) with a final observing night on July 30-31. During the week of impacts the 4".4 slit was aligned along the parallel of the impact sites (lat $=-44^{\circ}$ ). This allowed to search into immediate implications of each impact to the Jovian atmosphere at the time of event and to study the evolution of the older impact sites additionally present in the spectra. Starting on $23 \mathrm{July}$, we also observed the counterpart of the impact region in the northern hemisphere (lat $=+44^{\circ}$ ) and proceeded with a mapping of the entire planet. Data were recorded in several spectral regions between $2 \mu \mathrm{m}$ and $5 \mu \mathrm{m}$ with preference to the regions around the $\mathrm{H}_{3}^{+}$multiplet at $3.5 \mu \mathrm{m}$ and the $\mathrm{H}_{2} \mathrm{~S}(1)$ line at $2.12 \mu \mathrm{m}$. Fragments $\mathrm{B}, \mathrm{F}$ and $\mathrm{H}$ were observed at time of impact.

Before and during the impact of fragment $B$ on July 16 (0:21-3:58 UT) spectra were recorded in the $2.12 \mu \mathrm{m}$ range. The spectra do not show any effects of impact B to the Jovian atmosphere. However, they also cover impact site $A$ (approx. 5h after impact), where the emission of the $\mathrm{H}_{2} \mathrm{~S}(1)$ quadrupole line was detected. From the $\mathrm{H}_{2}$ observations recorded shortly after impact $\mathrm{F}$ on $18 \mathrm{July}, 2: 30 \mathrm{UT}$, we are able to distinguish between the impact sites $\mathrm{E}$ and $F$ that lie side by side very close together. The spectrum of $F$ is characterized 
by a strong featureless continuum, whereas the already evolved site $\mathrm{E}$ shows little continuum, but strong $\mathrm{H}_{2} \mathrm{~S}(1)$ line emission.

A strong emission of $\mathrm{CH}_{4}$ was detected in the $3.50-3.56 \mu \mathrm{m}$ range 13 minutes after impact $\mathrm{H}$ on $18 \mathrm{July}, 19: 46 \mathrm{UT}$, corresponding to 5 multiplets of the $\mathrm{CH}_{4} \nu_{3} \mathrm{P}$ band centered at $3.3 \mu \mathrm{m}$ (P16 to P20). In the first image, the peak intensity at the center of the emission corresponds to a rotational temperature of about $700 \mathrm{~K}$. The temporal evolution of this emission was monitored for 30 minutes while, over the first 15 minutes, its intensity decreased exponentially with a mean timescale of 3 minutes. Emissions of $\mathrm{H}_{3}^{+}(3.53 \mu \mathrm{m})$ were also detected over most of the impact sites several hours after the impacts. The evolution of these emissions could be followed until the end of the observational run.

After the impacts, between July 22 and 31,1994 , the evolution of the Jovian atmosphere was monitored in the $2.1 \mu \mathrm{m}$ and $3.5 \mu \mathrm{m}$ regions. From July 23 to 25 , the spectra recorded at $3.5 \mu \mathrm{m}$ show $\mathrm{H}_{3}^{+}$emissions in the northern hemisphere at $+44^{\circ}$ latitude that are clearly correlated to the impact sites. The emissions are not uniformly distributed, but are concentrated at the longitudes of the impact areas. They could result from the transfer of charged particles along the Jovian magnetic field lines. At $2.1 \mu \mathrm{m}$ clear evidence for enhanced emissions at the longitudes of the impact sites at $+44^{\circ}$ latitude was found only on July 23 . For a more detailed study Jupiter was mapped by scanning the planet along its rotational axis from pole to pole. Maps of $\mathrm{H}_{2}$ and $\mathrm{H}_{3}^{+}$ were constructed allowing the analysis of the spatial and temporal evolution of these species. The spatial distribution of the emissions shows a clear trend: the later after the impacts the more emission was present in the northern hemisphere, in particular in the northern polar region. The extremely strong emissions in the northern aurora might have led to a contamination of the spectra centered at $+44^{\circ}$ latitude. Thus, although there are still indications for the presence of northern counterparts at the longitude of the impacts sites at the end of the observational run, it seems that some of the emission is actually associated to enhanced auroral activity which extends to unusually low latitudes.

\title{
NEAR-INFRARED OBSERVATIONS OF THE ENCOUNTER OF COMET SHOEMAKER-LEVY 9 WITH JUPITER WITH THE ESO-GERMAN 2.2m TELESCOPE ON LA SILLA, CHILE
}

\author{
K. Jockers (MPI Aeronomy, Katlenburg-Lindau, Germany)
}

